\title{
CONSTRUCTION OF SPHERICAL CUBATURE FORMULAS USING LATTICES
}

\author{
P. DE LA HARPE, C. PACHE, AND B. VENKOV
}

\begin{abstract}
We construct cubature formulas on spheres supported by homothetic images of shells in some Euclidean lattices. Our analysis of these cubature formulas uses results from the theory of modular forms. Examples are worked out on $\mathbb{S}^{n-1}$ for $n=4,8,12,14,16,20,23$, and 24 , and the sizes of the cubature formulas we obtain are compared with the lower bounds given by Linear Programming.
\end{abstract}

\section{$\S 1$. INTRODUCTION}

For dimension $n \geq 2$ and a positive number $r$, let $\mathbb{S}_{r}^{n-1}$ denote the sphere given by the equation $\langle x \mid x\rangle=r$ in the Euclidean space $\mathbb{R}^{n}$ with the canonical scalar product $\langle\cdot \mid \cdot\rangle$, and let $\sigma$ denote the rotation-invariant measure on such a sphere, normalized by $\sigma\left(\mathbb{S}_{r}^{n-1}\right)=1$; we write $\mathbb{S}^{n-1}$ for $\mathbb{S}_{1}^{n-1}$. For an integer $t \geq 0$, a cubature formula of strength $t$ on $\mathbb{S}_{r}^{n-1}$ is a pair $(X, W)$, where $X$ is a finite subset of $\mathbb{S}_{r}^{n-1}$ and $W: \mathbb{S}_{r}^{n-1} \rightarrow \mathbb{R}_{>0}$ is a positive-valued function such that

$$
\sum_{x \in X} W(x) f(x)=\int_{\mathbb{S}_{r}^{n-1}} f(x) d \sigma(x)
$$

for every polynomial function $f: \mathbb{R}^{n} \rightarrow \mathbb{C}$ of degree at most $t$. The set $X$ is the support of the cubature formula $(X, W)$, the points in $X$ are its nodes, the values $W(x)$ are its weights, and the cardinality $|X|$ of $X$ is its size. A spherical t-design on $\mathbb{S}_{r}^{n-1}$ is a finite subset $X$ of $\mathbb{S}_{r}^{n-1}$ which, together with the constant weight (by necessity of value $|X|^{-1}$ ), is a cubature formula of strength $t$.

There are existence results according to which, for any dimension $n$ and strength $t$, there exist cubature formulas, and indeed spherical designs, of strength $t$ on $\mathbb{S}^{n-1}$. For cubature formulas, there are elementary arguments using general methods of convexity that give some upper bounds on the sizes (see 92 ); the existence of spherical designs is a particular case of the results of [SeyZas84, which do not provide meaningful size bounds. There are no known straightforward constructions, except for $n=2$, in which case, for any $t \geq 0$, the $(t+1)$ st roots of unity in $\mathbb{C}$ provide a spherical $t$-design on the circle, and for low values of $t$ in larger dimensions (the antipodal pairs, regular simplexes, and regular hyperoctahedra are respectively spherical 1-, 2-, and 3-designs of the lowest possible sizes).

It is therefore a natural question to ask for more explicit constructions. Some have been given in terms of orbits of finite groups on the sphere (see HarPac04), and by other various methods (see Bajn91 and later papers by Bajnok, and also Kupe06a, Kupe06b, and [HadSlo96] for $\mathbb{S}^{2}$ ).

2000 Mathematics Subject Classification. Primary 65D32, 05B30; Secondary 11F11, 11H06.

Key words and phrases. Cubature formula, modular lattice, modular form, spherical $t$-design.

The authors acknowledge support from the Swiss National Science Foundation. 
Our aim in the present paper is to describe constructions involving shells (or layers) of integral lattices in $\mathbb{R}^{n}$. They are elaborations of constructions which can also be found in MartV01], Pach05], and [HarPac05].

Let $E$ be a discrete subset of $\mathbb{R}^{n}$. For any $m>0$, denote by $E_{m}$ the shell $E \cap \mathbb{S}_{m}^{n-1}$. If $m_{1}, \ldots, m_{r}$ are pairwise distinct positive numbers such that each shell $E_{m_{j}}$ is nonempty, the union of the sets $\frac{1}{\sqrt{m_{j}}} E_{m_{j}}$ is a finite subset (possibly with multiplicities) of the unit sphere in $\mathbb{R}^{n}$. The spherical designs and cubature formulas that appear below have supports of this kind, where $E$ is either an integral lattice in $\mathbb{R}^{n}$ or a union of lattices; observe that they are all antipodal, namely, that $X=-X$ and $W(x)=W(-x)$ for all $x \in X$.

Results on $\mathbb{S}^{3}$. Let us describe some examples provided by this method for $n=4$, and compare them to known lower bounds for sizes and to other known examples. For $n \geq 4$, the root lattice of type $\mathbf{D}_{n}$ is the integral lattice

$$
D_{n}=\left\{x \in \mathbb{Z}^{n} \mid x_{1}+\cdots+x_{n} \equiv 0(\bmod 2)\right\} .
$$

(We denote root systems by $\mathbf{D}_{n}$ with bold letters and lattices by $D_{n}$ with thin letters.) It is important for our computations that $D_{4}$ is a 2-modular lattice (the definition is recalled in $\$ 4$ below).

For $n \geq 5, n \neq 7$, it is known that each shell $\left(D_{n}\right)_{2 m}$ is a spherical 3-design which is in general not of strength 4 ; for $n=4$, each shell $\left(D_{4}\right)_{2 m}$ is a spherical 5-design which is in general not of strength 6 (as was checked in Pach05, it is never of strength 6 for $2 m \leq 1200)$. The sizes of the shells of the root lattice of type $\mathbf{D}_{4}$ are given by the coefficients of the theta series

$$
\begin{aligned}
\Theta_{D_{4}} & =\sum_{m \geq 0}\left|\left(D_{4}\right)_{2 m}\right| q^{2 m}=\sum_{m \geq 0} r_{4}(2 m) q^{2 m} \\
& =1+24 q^{2}+24 q^{4}+96 q^{6}+24 q^{8}+144 q^{10}+96 q^{12}+192 q^{14}+\cdots,
\end{aligned}
$$

where $r_{4}(2 m)$ denotes the number of ways of writing $2 m$ as a sum of four squares.

The dual $D_{4}^{*}=\left\{y \in \mathbb{R}^{n} \mid\left\langle y \mid D_{4}\right\rangle \subset \mathbb{Z}\right\}$ is similar to $D_{4}$, and the renormalized dual $D_{4}^{\prime}=\sqrt{2} D_{4}^{*}$ is isometric to $D_{4}$; moreover, the intersection $D_{4} \cap D_{4}^{\prime}$ is reduced to $\{0\}$, since the nonzero coordinates of the vertices in $D_{4}^{\prime}$ are never in $\mathbb{Z}$. For our analysis, we use some invariant theory of the finite group $\operatorname{Aut}\left(D_{4} \cup D_{4}^{\prime}\right)$.

Theorem $D_{4}$. Set $E=D_{4} \cup D_{4}^{\prime}$.

(i) For any even integer $2 m \geq 2$, the shell $E_{2 m}$ is a spherical 7-design; in particular, $E_{2}$ is a spherical 7-design of size 48 in $\mathbb{S}_{2}^{3}$.

(ii) The two shells $E_{2}, E_{6}$ provide the support $X=\frac{1}{\sqrt{2}} E_{2} \cup \frac{1}{\sqrt{6}} E_{6}$ of a cubature formula of strength 11 and size 240.

(iii) Similarly, $\frac{1}{\sqrt{2}} E_{2} \cup \frac{1}{\sqrt{6}} E_{6} \cup \frac{1}{\sqrt{10}} E_{10}$ is the support of a cubature formula of strength 15 and size 528 .

Claim (i) for $E_{2}$ appears already in $\S 6$ of GoeSei81, where the authors observe that $E_{2}$ is an orbit of the Weyl group of type $\mathbf{F}_{4}$. Claim (i) of Theorem $E_{8}$ below and the analogous result involving the two shortest shells of a Leech lattice appear also in GoeSei81.

For comparison, here are some known results concerning the 3-sphere.

Dickson reports on a formula due to Liouville (1859) which essentially shows that the system of roots $\left(D_{4}\right)_{2}$ of type $\mathbf{D}_{4}$ is a spherical 5-design, a formula due to Kempner (1912) which essentially shows that $\left(D_{4} \cup D_{4}^{\prime}\right)_{2}$ is a spherical design of strength 7 , and a formula due to Schur (1909) related to a cubature formula of strength 11 and size 144 . See [Dick19, pp. 717-724]. 
There is a formula of strength 7 and size 46 described in HadSlo94].

There is an exceptional regular polytope in dimension four known as the 600-cell. It has 120 vertices that constitute a spherical 11-design; it is an orbit of a Coxeter group of type $\mathbf{H}_{4}$. Any orbit of this group is a spherical design of strength 11, but there is a special orbit of size 1440 and of strength 19; see GoeSei81, §5]. Another cubature formula of the same strength 19 and of size 720 appears in Sali75: its support is the union of the 120 vertices and the 600 centers of the faces of the 600 -cell.

The best lower bounds known to us for the sizes of cubature formulas in $\mathbb{S}^{3}$ are listed in Tables 6 and 7 of $₫ 7$

Results on $\mathbb{S}^{7}$. Let $\Gamma_{8}$ be a Korkin-Zolotarev lattice, namely, an even unimodular lattice of dimension 8 (uniquely defined up to isometry by these properties). Such a lattice is generated by a root system of type $\mathbf{E}_{8}$. The sizes of its shells are given by the coefficients of the theta series

$$
\begin{aligned}
\Theta_{\Gamma_{8}} & =\sum_{m \geq 0}\left|\left(\Gamma_{8}\right)_{2 m}\right| q^{2 m}=240 \sum_{m \geq 0} \sigma_{3}(m) q^{2 m} \\
& =1+240 q^{2}+2160 q^{4}+6720 q^{6}+17520 q^{8}+\cdots
\end{aligned}
$$

where $\sigma_{3}(m)=\sum_{d \mid m} d^{3}$.

It is known that each shell $\left(\Gamma_{8}\right)_{2 m}$ is a spherical 7-design which is in general not of strength 8 . Indeed, the shell $\left(\Gamma_{8}\right)_{2 m}$ would be a design of strength 8 if and only if the Ramanujan function $\tau$ would vanish at $m$; it is a conjecture of Lehmer [Lehm47] that $\tau(m) \neq 0$ for all $m \geq 1$, and this has been checked for $m \leq 10^{15}$ Serr85; more on this in Pach05] and in Item 1.16 of [HarPac05].

Theorem $E_{8}$. Set $E=\Gamma_{8}$.

(i) The two shells $E_{2}, E_{4}$ provide the support $X=\frac{1}{\sqrt{2}} E_{2} \cup \frac{1}{2} E_{4}$ of a cubature formula of strength 11 and size 2400 .

(ii) Similarly, $\frac{1}{\sqrt{2}} E_{2} \cup \frac{1}{\sqrt{6}} E_{6} \cup \frac{1}{2 \sqrt{2}} E_{8}$ is the support of a cubature formula of strength 13 and size 24240 .

(iii) Similarly, $\frac{1}{\sqrt{2}} E_{2} \cup \frac{1}{2} E_{4} \cup \frac{1}{\sqrt{6}} E_{6} \cup \frac{1}{2 \sqrt{2}} E_{8}$ is the support of a cubature formula of strength 15 and size 26400 .

The largest known lower bounds for the sizes of cubature formulas on $\mathbb{S}^{7}$ are given in Table 8 in 87 .

Dimensions 12, 14, 16, 20, 23, and 24. There are similar constructions on $\mathbb{S}^{23}$ in terms of a Leech lattice, an even unimodular lattice $\Lambda$ of dimension 24 with empty shell $\Lambda_{2}$ (uniquely defined up to isometry by these properties).

We also describe constructions with shells of

- the 3-modular Coxeter-Todd lattice $K_{12}$,

- the 3-modular Quebbemann lattice $Q_{14}$,

- the 2-modular Barnes-Wall lattice $B W_{16}$,

- the 2-modular Nebe lattices $N_{20}$ (three of them),

- the 3-modular Nebe lattice $N_{24}$, and

- the shorter Leech lattice $O_{23}$ (which is up to isometry a unique unimodular integral lattice $\Lambda$ in $\mathbb{R}^{23}$ with $\Lambda_{1}=\Lambda_{2}=\varnothing$ ).

Constructions with one shell of these lattices were already described in BacVen01. Our results appear in Tables 9 to 14 in $\$ 7$ 


\section{$\S 2$. BOUNDS ON THE SIZES OF CUBATURE FORMULAS}

For fixed values of $n$ and $t$, we are interested in constructing cubature formulas on $\mathbb{S}_{r}^{n-1}$ of strength $t$ of small size; it is therefore useful to estimate the minimal size that such a cubature formula can have. We recall in this section some general results on this subject.

Let $\mathrm{b}(n, t)$ be the minimal size of a spherical cubature formula on $\mathbb{S}_{r}^{n-1}$ of strength $t$.

Linear Programming bound (see [DeGoSe77, Theorem 5.10] and Yudi97]). Let $\mathrm{Q}^{(k)}$, $k \in \mathbb{Z}_{\geq 0}$, be the orthogonal polynomials for the scalar product

$$
\langle f \mid g\rangle:=\int_{-1}^{1} f(t) g(t)\left(1-t^{2}\right)^{(n-3) / 2} d t
$$

normalized to $\left\langle\mathrm{Q}^{(k)} \mid \mathrm{Q}^{(k)}\right\rangle=\mathrm{Q}^{(k)}(1)$. For a continuous function $F:[-1,1] \rightarrow \mathbb{R}$, we define numbers $F_{k} \in \mathbb{R}$ by

$$
F_{k}=\frac{\left\langle F \mid \mathrm{Q}^{(k)}\right\rangle}{\mathrm{Q}^{(k)}(1)}
$$

so that $F=\sum_{k \geq 0} F_{k} \mathrm{Q}^{(k)}$. Set

$$
M(n, t):=\left\{\begin{array}{l|l}
F:[-1,1] \rightarrow \mathbb{R} \text { continuous } & \begin{array}{l}
F(u) \geq 0 \text { for } u \in[-1,1], F \neq 0, \\
\text { and } F_{k} \leq 0 \text { for } k>t
\end{array}
\end{array}\right\}
$$

then $\mathrm{b}(n, t) \geq F(1) / F_{0}$ for every $F \in M(n, t)$. In other words,

$$
\mathrm{b}_{\mathrm{LP}}(n, t):=\sup _{F \in M(n, t)} \frac{F(1)}{F_{0}}
$$

is a lower bound for $\mathrm{b}(n, t)$. Moreover, it is a consequence of NikNik02, Theorems I and 2.7] that there exists a polynomial function $F \in M(n, t)$ for which $\mathrm{b}_{\mathrm{LP}}(n, t)=$ $F(1) / F_{0}$.

When $t$ is odd, we show the following.

1. Proposition. Let $t=2 s+1$, and let

$$
\widehat{M}(n, t):=\{F \in M(n, t) \mid F(-u)=F(u)\} .
$$

Then

$$
\mathrm{b}_{\mathrm{LP}}(n, t)=\sup _{F \in \widehat{M}(n, t)} \frac{2 F(1)}{F_{0}}
$$

Proof. Let $F \in M(n, t)$; setting $\widehat{F}(u)=(F(u)+F(-u)) / 2$, we have $\widehat{F} \in \widehat{M}(n, t)$. Set $F^{*}(u)=(1+u) \widehat{F}(u)$. It can be checked that

$$
F^{*} \in M(n, t) \text {. }
$$

Yet $2 \widehat{F}(1)=F(1)+F(-1) \geq F(1)$ and $F_{0}=\widehat{F}_{0}=F_{0}^{*}$; therefore,

$$
\frac{F(1)}{F_{0}} \leq \frac{2 \widehat{F}(1)}{\widehat{F}_{0}}=\frac{F^{*}(1)}{F_{0}^{*}} .
$$

The result follows.

In general, the exact value of $\mathrm{b}_{\mathrm{LP}}(n, t)$ is not known. The following bounds are obtained by choosing a particular $F$ in $M(n, t)$. 
Delsarte (or Fisher-type) bound (see DeGoSe77, Theorems 5.11 and 5.12]). Choose

$$
F(u):=\left(\sum_{k=0}^{s} \mathrm{Q}^{(k)}(u)\right)^{2}
$$

if $t=2 s$, and

$$
F(u):=(1+u)\left(\sum_{j=0}^{[s / 2]} \mathrm{Q}^{(s-2 j)}(u)\right)^{2}
$$

if $t=2 s+1$. We obtain the bounds

$$
\begin{aligned}
\mathrm{b}_{\mathrm{D}}(n, 2 s) & =\left(\begin{array}{c}
n+s-1 \\
n-1
\end{array}\right)+\left(\begin{array}{c}
n+s-2 \\
n-1
\end{array}\right), \\
\mathrm{b}_{\mathrm{D}}(n, 2 s+1) & =2\left(\begin{array}{c}
n+s-1 \\
n-1
\end{array}\right) .
\end{aligned}
$$

Moreover, it is known that if $n \geq 3$, the equality $\mathrm{b}(n, t)=\mathrm{b}_{\mathrm{D}}(n, t)$ is possible only for some values of $(n, t)$; see BanDam79, BanDam80, and BaMuVe02. There also exists a criterion to decide when $\mathrm{b}_{\mathrm{D}}(n, t)=\mathrm{b}_{\mathrm{LP}}(n, t)$; see [NikNik02, Theorem I].

Yudin bound (see [Yudi97]). Another choice of $F$ gives the bound

$$
\mathrm{b}_{\mathrm{Y}}(n, t)=\frac{\int_{-1}^{1}\left(1-u^{2}\right)^{(n-3) / 2} d u}{\int_{\gamma}^{1}\left(1-u^{2}\right)^{(n-3) / 2} d u}
$$

where $\gamma$ is the largest root of the polynomial $\left(\mathrm{Q}^{(t+1)}\right)^{\prime}$.

Special cases. For some values of $(n, t)$, it is possible to compute the exact value of $\mathrm{b}(n, t)$. For example, in Andr00] it was shown that $\mathrm{b}(4,11)=\mathrm{b}_{\mathrm{LP}}(4,11)=120$.

Numerical estimate of the Linear Programming bound. For fixed values of $(n, t)$, $t=2 s+1$, the following procedure gives an estimate of the Linear Programming bound $\mathrm{b}_{\mathrm{LP}}(n, t)$. (There is a similar procedure when $t$ is even.)

- Choose a degree $d \geq s$ and a finite subset $A \subseteq[0,1]$ of well-distributed points; for example, $A=\{i / N \mid i=0,1, \ldots, N\}$ with $N$ large.

- By linear programming, among all polynomials $F$ satisfying

$$
F=1+\sum_{i=1}^{d} F_{k} \mathrm{Q}^{(2 k)}, \quad F(u) \geq 0 \text { for } u \in A, \quad F_{k} \leq 0 \text { for } k>t,
$$

find a polynomial minimizing $F(1)$; denote it by $G$.

- Set $\epsilon:=-\inf _{x \in[0,1]} G(x) \geq 0$. The polynomial $\widetilde{G}:=G+\epsilon$ is in the set $\widehat{M}(n, t)$, and we have the estimate

$$
\frac{2 \widetilde{G}(1)}{\widetilde{G}_{0}}=\frac{2(G(1)+\epsilon)}{1+\epsilon} \leq \mathrm{b}_{\mathrm{LP}}(n, t) .
$$

Note that we also have $\mathrm{b}_{\mathrm{LP}}^{(d)}(n, t) \leq 2 G(1)$, where

$$
\mathrm{b}_{\mathrm{LP}}^{(d)}(n, t):=\sup _{\substack{F \in M(n, t) \\ \operatorname{deg} f \leq d}} \frac{F(1)}{F_{0}} .
$$


Yet, if $d$ is sufficiently large and $A$ sufficiently dense, then $2 \widetilde{G}(1) / \widetilde{G}_{0}$ is a good approximation of $\mathrm{b}_{\mathrm{LP}}(n, t)$. In practice, we first apply the procedure with a relatively large $d$ and a relatively small set $A$; we observe that $G_{k}=0$ for $k>d_{0}$. Then we apply the procedure with $d=d_{0}$ and with a larger set $A$.

The procedure described above imitates that used in [ConSlo99, Chapter 13] to compute bounds for kissing numbers.

We end this section with another kind of a bound. Set

$$
\mathrm{B}(n, t):=\left(\begin{array}{c}
n+t-1 \\
n-1
\end{array}\right)+\left(\begin{array}{c}
n+t-2 \\
n-1
\end{array}\right) .
$$

Note that $\mathrm{B}(n, t)=\mathrm{b}_{\mathrm{D}}(n, 2 t)$. This number is the dimension of the space of restrictions to the sphere of the polynomial functions of degree at most $t$.

2. Proposition. For every $n$ and $t$, there exist cubature formulas with at most $\max \{1$, $\mathrm{B}(n, t)-1\}$ nodes.

3. Proposition. Let $(X, W)$ be a cubature formula on $\mathbb{S}_{r}^{n-1}$ of strength $t$. Then there exists a subset $X^{\prime} \subseteq X$ and a weight function $W^{\prime}: X^{\prime} \rightarrow \mathbb{R}_{>0}$ such that $\left|X^{\prime}\right| \leq \mathrm{B}(n, t)$ and $\left(X^{\prime}, W^{\prime}\right)$ is a cubature formula of degree $t$.

See HarPac05, Proposition 2.6]. The second proposition is a consequence of the proof of the first and provides an algorithm for computing $\left(X^{\prime}, W^{\prime}\right)$ starting with $(X, W)$.

Note however that these propositions fail for spherical designs (the case of constant weights).

\section{§3. HARMONIC POLYNOMIALS}

It is sufficient to check condition $(\mathrm{CF})$ in the definition of cubature formulas for harmonic polynomials only. Recall that a smooth function $f$ on $\mathbb{R}^{n}$ is harmonic if $\Delta f=0$, where $\Delta=\sum_{i=1}^{n}\left(\partial / \partial x_{i}\right)^{2}$ is the usual Laplacian. We denote by $\mathcal{H}^{(k)}\left(\mathbb{R}^{n}\right)$ the space of harmonic polynomial functions $\mathbb{R}^{n} \rightarrow \mathbb{C}$ that are homogenous of degree $k$. It is a classical fact that the space of restrictions to $\mathbb{S}^{n-1}$ of homogenous polynomials of degree at most $t$ coincides with the space of restrictions of the direct sum $\bigoplus_{k=0}^{t} \mathcal{H}^{(k)}\left(\mathbb{R}^{n}\right)$. This implies the following well-known criterion; see for example VenMar01, Theorem 3.2] (details are written there for spherical designs, but the proof carries over to cubature formulas).

4. Proposition. Let $X$ be a finite subset of some sphere $\mathbb{S}_{m}^{n-1}$ in $\mathbb{R}^{n}$, let $W: X \rightarrow \mathbb{R}_{>0}$ be a weight function, and let $t \geq 0$ be an integer. Then $(X, W)$ is a cubature formula of strength $t$ on $\mathbb{S}_{m}^{n-1}$ if and only if $\sum_{x \in X} W(x)=1$ and

$$
\sum_{x \in X} W(x) P(x)=0 \quad \text { for all } P \in \mathcal{H}^{(k)}\left(\mathbb{R}^{n}\right), 1 \leq k \leq t .
$$

Let $\mathrm{O}(n)$ be the orthogonal group of the Euclidean space $\mathbb{R}^{n}$; this group acts by isometries on the sphere $\mathbb{S}_{r}^{n-1}$. Moreover, $\mathrm{O}(n)$ acts naturally on $\mathcal{H}^{(k)}\left(\mathbb{R}^{n}\right)$ by $g \cdot f=$ $f \circ g^{-1}$.

Whenever a group $G$ acts on a space $V$, we denote by $V^{G}$ the subspace of all elements of $V$ fixed by $G$.

Proposition 4 above can be refined in the case where the cubature formula is invariant by a finite subgroup of $G$. Namely, we have the following result, known as Sobolev's theorem ([Sobo62], see also [Sobo96, Chapter 2, §2, Theorem 2.3]).

5. Proposition. Let $X$ be a finite subset of $\mathbb{S}_{r}^{n-1}$, and let $W: X \rightarrow \mathbb{R}_{>0}$ be a weight function. Let $G$ be a finite subgroup of $\mathrm{O}(n)$ leaving $(X, W)$ invariant, that is,

$$
g x \in X \text { and } W(g x)=W(x) \text { for all } x \in X, g \in G .
$$


Then $(X, W)$ is a cubature formula of strength $t$ if and only if $\sum_{x \in X} W(x)=1$ and

$$
\sum_{x \in X} W(x) P(x)=0 \quad \text { for all } P \in \mathcal{H}^{(k)}\left(\mathbb{R}^{n}\right)^{G}, 1 \leq k \leq t .
$$

Proof. For $P \in \mathcal{H}^{(k)}\left(\mathbb{R}^{n}\right)$, let $P^{G}:=|G|^{-1} \sum_{g \in G} g \cdot f$. On the one hand, the $G$-invariance of $(X, W)$ implies

$$
\sum_{x \in X} W(x) P^{G}(x)=\sum_{x \in X} W(x) P(x),
$$

and, on the other hand, the $G$-invariance of the Lebesgue measure implies

$$
\int_{\mathbb{S}_{r}^{n-1}} P^{G}(x) d \sigma(x)=\int_{\mathbb{S}_{r}^{n-1}} P(x) d \sigma(x) .
$$

The result follows from Proposition 4.

\section{$\S 4$. MOdULAR FORMS}

This section is a reminder from BacVen01. Consider a lattice $\Lambda$ in $\mathbb{R}^{n}$ and a homogeneous harmonic polynomial $P \in \mathcal{H}^{(k)}\left(\mathbb{R}^{n}\right)$ of degree $k$. The theta series of $\Lambda$ with harmonic coefficient $P$ is the formal power series $\Theta_{\Lambda, P}$ defined by

$$
\Theta_{\Lambda, P}:=\sum_{x \in \Lambda} P(x) q^{\langle x \mid x\rangle}=P(0)+\sum_{m>0}\left(\sum_{x \in \Lambda_{m}} P(x)\right) q^{m} .
$$

In case $P=1$, we write simply $\Theta_{\Lambda}$. Set

$$
q:=e^{i \pi z}, \quad z \in \mathbb{H},
$$

where $\mathbb{H}=\{z \in \mathbb{C} \mid \operatorname{Im} z>0\}$ is the Poincaré half-plane; the theta series $\Theta_{\Lambda, P}$ converges uniformly on every compact subset of $\mathbb{H}$ and defines consequently a holomorphic function on $\mathbb{H}$. If the lattice $\Lambda$ satisfies appropriate conditions, this function is a modular form of weight $\omega$, and we can use results on modular forms for the computation of the theta series.

First, we describe the class of lattices that play the most important role in the sequel. A lattice $\Lambda$ in $\mathbb{R}^{n}$ is even if $\langle x \mid x\rangle \in 2 \mathbb{Z}$ for all $x \in \Lambda$; such a lattice is contained in its dual $\Lambda^{*}=\left\{y \in \mathbb{R}^{n} \mid\langle\Lambda \mid y\rangle \subseteq \mathbb{Z}\right\}$, and there are integers $\ell \geq 1$ such that $\sqrt{\ell} \Lambda^{*}$ is again even ( $\ell$ is not unique because if $\sqrt{\ell} \Lambda^{*}$ is even, so is $\sqrt{k^{2} \ell} \Lambda^{*}$ for $k \geq 1$ ). For given integers $n$ and $\ell$, denote by $\mathcal{L} a t_{n}(\ell)$ the class of even lattices $\Lambda$ such that $\Lambda^{\prime}:=\sqrt{\ell} \Lambda^{*}$ is even and $\operatorname{det}\left(\Lambda^{\prime}\right)=\operatorname{det}(\Lambda)$; the latter condition implies that $\operatorname{det}(\Lambda)=\ell^{n / 2}$. Clearly, if $\Lambda \in \operatorname{Lat}_{n}(\ell)$, then $\Lambda^{\prime} \in \mathcal{L a t}_{n}(\ell)$. A lattice $\Lambda \in \mathcal{L} a t_{n}(\ell)$ is $\ell$-modular if $\Lambda^{\prime}$ is equivalent to $\Lambda$.

Now, we describe the modular forms associated with these lattices. For a nonnegative integer $\omega$ and $\epsilon \in\{+,-\}$, we define

$$
\widehat{\mathcal{M}}_{\omega}^{\epsilon}(\ell)
$$

as the space of holomorphic functions $f: \mathbb{H} \rightarrow \mathbb{C}$ that satisfy

$$
\begin{gathered}
f(z+1)=f(z), \\
f\left(-\frac{1}{\ell z}\right)=\epsilon\left(\frac{i}{\sqrt{\ell} z}\right)^{\omega} f(z)
\end{gathered}
$$

for all $z \in \mathbb{H}$, and that are holomorphic at infinity, i.e., bounded on $\left\{z \in \mathbb{H} \mid \operatorname{Im} z>y_{0}\right\}$ for $y_{0}>0$. This means that $f$ can be written as

$$
f(z)=\sum_{m \geq 0} a_{m} q^{2 m}, \quad q=e^{i \pi z} .
$$


Let $f(\infty):=\lim _{\operatorname{Im} z \rightarrow \infty} f(z)=a_{0}$. We define

$$
{ }^{0} \widehat{\mathcal{M}}_{\omega}^{\epsilon}(\ell)=\left\{f \in \widehat{\mathcal{M}}_{\omega}^{\epsilon}(\ell) \mid f(\infty)=0\right\} .
$$

We give here the classical terminology. A modular form of weight $\omega \geq 0$ for a discrete group $\Gamma \subseteq \mathrm{SL}_{2}(\mathbb{R})$ and a character $\chi: \Gamma \rightarrow \mathbb{R}$ is a holomorphic function $f: \mathbb{H} \rightarrow \mathbb{C}$ that is holomorphic at infinity and satisfies

$$
\frac{\chi\left(\left(\begin{array}{ll}
a & b \\
c & d
\end{array}\right)\right)}{(c z+d)^{\omega}} f\left(\frac{a z+b}{c z+d}\right)=f(z) \quad \text { for all }\left(\begin{array}{ll}
a & b \\
c & d
\end{array}\right) \in \Gamma .
$$

A parabolic form is a modular form $f$ that is zero at infinity, namely, such that $f(\infty)=0$.

For a positive integer $\ell$, let $\Gamma_{*}(\ell)$ be the subgroup of $\mathrm{SL}_{2}(\mathbb{R})$ generated by

$$
\Gamma_{0}(\ell)=\left\{\left(\begin{array}{ll}
a & b \\
c & d
\end{array}\right) \in \mathrm{SL}_{2}(\mathbb{Z}) \mid c \equiv 0 \bmod \ell\right\} \quad \text { and } \quad t_{\ell}=\left(\begin{array}{cc}
0 & 1 / \sqrt{\ell} \\
-\sqrt{\ell} & 0
\end{array}\right) .
$$

For any integer $s$, let $\chi_{s}$ be the multiplicative character of $\Gamma_{*}(\ell)$ defined by

$$
\begin{aligned}
\chi_{s}\left(\left(\begin{array}{ll}
a & b \\
c & d
\end{array}\right)\right) & =\left(\frac{(-\ell)^{s}}{d}\right) \quad \text { for }\left(\begin{array}{ll}
a & b \\
c & d
\end{array}\right) \in \Gamma_{0}(\ell), \\
\chi_{s}\left(t_{\ell}\right) & =i^{s},
\end{aligned}
$$

where (-) denotes the Kronecker symbol. Note that $\chi_{s}$ depends only on the class of $s$ modulo 4. Denote by $\mathcal{M}_{\omega}^{+}(\ell)$ the space of modular forms of weight $\omega$ for the group $\Gamma_{*}(\ell)$ and the character $\chi_{\omega}$, and by $\mathcal{M}_{\omega}^{-}(\ell)$ the space of modular forms of weight $\omega$ for the same group and the character $\chi_{\omega+2}$; denote by ${ }^{0} \mathcal{M}_{\omega}^{ \pm}(\ell)$ the space of the corresponding parabolic forms.

Note that equations $(*)$ say that $\widehat{\mathcal{M}}_{\omega}^{+}(\ell)$ (respectively, $\widehat{\mathcal{M}}_{\omega}^{-}(\ell)$ ) is the space of modular forms of weight $\omega$ for the subgroup generated by

$$
T=\left(\begin{array}{ll}
1 & 1 \\
0 & 1
\end{array}\right) \quad \text { and } \quad S_{\ell}=\left(\begin{array}{cc}
0 & 1 / \sqrt{\ell} \\
-\sqrt{\ell} & 0
\end{array}\right)
$$

and for the character $\chi_{\omega}$ (respectively, $\chi_{\omega+2}$ ).

6. Lemma. For $\ell \in\{1,2,3\}$, the group $\Gamma_{*}(\ell)$ is generated by $T$ and $S_{\ell}$. In particular, for these values of $\ell$ we have

$$
\widehat{\mathcal{M}}_{\omega}^{\epsilon}(\ell)=\mathcal{M}_{\omega}^{\epsilon}(\ell) \quad \text { and } \quad{ }^{0} \widehat{\mathcal{M}}_{\omega}^{\epsilon}(\ell)={ }^{0} \mathcal{M}_{\omega}^{\epsilon}(\ell) .
$$

Proof. Let $\ell \in\{1,2,3\}$ and $\gamma \in\left(\begin{array}{ll}a & b \\ c & d\end{array}\right) \in \Gamma_{*}(\ell)$. We show, by induction on $c^{2} \in \mathbb{N}$, that $\gamma \in\left\langle T, S_{\ell}\right\rangle$, where $\left\langle T, S_{\ell}\right\rangle$ denotes the group generated by $T$ and $S_{\ell}$. Observe that $S_{\ell}^{2}=\left(\begin{array}{cc}-1 & 0 \\ 0 & -1\end{array}\right)$.

If $c=0$, then $\gamma=\left(\begin{array}{cc}1 & m \\ 0 & 1\end{array}\right)=T^{m}$ for an integer $m$; thus $\gamma \in\left\langle T, S_{\ell}\right\rangle$.

If $c \neq 0$, let $k \in \mathbb{Z}$ be such that $|d+k c| \leq|c / 2|$. We have

$$
\gamma T^{k} S_{\ell}=\left(\begin{array}{ll}
-(b+k a) \sqrt{\ell} & a / \sqrt{\ell} \\
-(d+k c) \sqrt{\ell} & c / \sqrt{\ell}
\end{array}\right),
$$

and $(-(d+k c) \sqrt{\ell})^{2} \leq c^{2}(\ell / 4)<c^{2}$. By induction, $\gamma T^{k} S_{\ell} \in\left\langle T, S_{\ell}\right\rangle$, whence $\gamma \in$ $\left\langle T, S_{\ell}\right\rangle$.

7. Proposition. Let $\Lambda \in \mathcal{L} a t_{n}(\ell)$, where $\ell$ is a positive integer. Then

$$
\begin{aligned}
& \Theta_{\Lambda}+\Theta_{\Lambda^{\prime}} \in \mathcal{M}_{n / 2}^{+}(\ell), \\
& \Theta_{\Lambda}-\Theta_{\Lambda^{\prime}} \in{ }^{0} \mathcal{M}_{n / 2}^{-}(\ell) .
\end{aligned}
$$


Let, moreover, $P \in \mathcal{H}^{(2 h)}\left(\mathbb{R}^{n}\right), h \geq 1$. In this case, if $2 h \equiv 0 \bmod 4$, then

$$
\begin{aligned}
& \Theta_{\Lambda, P}+\Theta_{\Lambda^{\prime}, P} \in{ }^{0} \mathcal{M}_{n / 2+2 h}^{+}(\ell), \\
& \Theta_{\Lambda, P}-\Theta_{\Lambda^{\prime}, P} \in{ }^{0} \mathcal{M}_{n / 2+2 h}^{-}(\ell),
\end{aligned}
$$

and if $2 h \equiv 2 \bmod 4$, then

$$
\begin{aligned}
& \Theta_{\Lambda, P}+\Theta_{\Lambda^{\prime}, P} \in{ }^{0} \mathcal{M}_{n / 2+2 h}^{-}(\ell), \\
& \Theta_{\Lambda, P}-\Theta_{\Lambda^{\prime}, P} \in{ }^{0} \mathcal{M}_{n / 2+2 h}^{+}(\ell) .
\end{aligned}
$$

Partial proof. We give the proof only for $\ell \in\{1,2,3\}$. The general case is more complicated: see Ebel94, §3.1] together with VenMar01, Chapter 2]. ${ }^{1}$

It is straightforward that the theta series involved are holomorphic in $\mathbb{H}$ and holomorphic at infinity. Now, by Lemma 6 , it suffices to check that the theta series satisfy equations $(*)$.

Since $\Lambda$ and $\Lambda^{\prime}$ are even, we have clearly $\Theta_{L, P}(z+1)=\Theta_{L, P}(z)$ for $L=\Lambda$ or $\Lambda^{\prime}$. Now, as a direct consequence of the Poisson summation formula (see for example Ebel94, Proposition 3.1, p. 87]), for any lattice $L$ of rank $n$ and any $P \in \mathcal{H}^{(2 h)}\left(\mathbb{R}^{n}\right)$ we have

$$
\Theta_{L^{*}, P}(z)=(\operatorname{det} L)^{1 / 2}(-1)^{h}(i / z)^{n / 2+2 h} \Theta_{L, P}(-1 / z) .
$$

From this formula, we deduce that, for $L=\Lambda$ or $\Lambda^{\prime}$,

$$
\Theta_{L^{\prime}, P}(z)=(-1)^{h}(i / \sqrt{\ell} z)^{n / 2+2 h} \Theta_{L, P}(-1 / \ell z) .
$$

The result follows.

Let

$$
\begin{gathered}
\mathcal{M}^{+}(\ell):=\bigoplus_{\omega \geq 0} \mathcal{M}_{\omega}^{+}(\ell), \\
{ }^{0} \mathcal{M}^{+}(\ell):=\bigoplus_{\omega>0}^{0} \mathcal{M}_{\omega}^{+}(\ell), \quad{ }^{0} \mathcal{M}^{-}(\ell):=\bigoplus_{\omega>0}{ }^{0} \mathcal{M}_{\omega}^{-}(\ell) .
\end{gathered}
$$

These are $\mathcal{M}^{+}(\ell)$-modules graded by the weight. For some values of $\ell$, the structure of these algebras is known (see the theorem below).

Remark. In general, the numerical subscript of modular forms given below indicates the double of its weight; for example, $\Delta_{24} \in{ }^{0} \mathcal{M}_{12}(1)$ is of weight 12 , and $\Theta_{\mathbf{E}_{8}} \in \mathcal{M}_{4}$ (1) is of weight 4 . We have made an exception for the Eisenstein series $E_{(k)}$, for which we have retained the traditional notation.

8. Theorem. Let $\ell$ be 1 or a prime number such that $\ell+1$ divides 24 . Let $k_{0}, k_{1}$, and $k_{2}$ be given by

$$
\begin{gathered}
k_{0}= \begin{cases}4 & \text { if } \ell=1, \\
2 & \text { if } \ell \equiv 1 \text { or } 2 \bmod 4, \text { and } \ell \neq 1, \\
1 & \text { if } \ell \equiv 3 \bmod 4,\end{cases} \\
k_{1}=\frac{24}{\ell+1}, \quad k_{2}=k_{0}+k_{1}+2 .
\end{gathered}
$$

\footnotetext{
${ }^{1}$ Note however that our examples of constructions of cubature formulas in $\sqrt[6]{6}$ involve lattices in $\operatorname{Lat}_{n}(\ell)$ for $\ell \in\{1,2,3\}$ only.
} 
Then

$$
\begin{aligned}
\mathcal{M}^{+}(\ell) & =\mathbb{C}\left[\theta_{2 k_{0}}, \Delta_{2 k_{1}}\right], \\
{ }^{0} \mathcal{M}^{+}(\ell) & =\Delta_{2 k_{1}} \mathbb{C}\left[\theta_{2 k_{0}}, \Delta_{2 k_{1}}\right], \\
{ }^{0} \mathcal{M}^{-}(\ell) & =\Phi_{2 k_{2}} \mathbb{C}\left[\theta_{2 k_{0}}, \Delta_{2 k_{1}}\right],
\end{aligned}
$$

where

$$
\begin{aligned}
\theta_{2 k_{0}}=\Theta_{L_{0}} \in & \mathcal{M}_{k_{0}}^{+}(\ell) \text { with } L_{0} \in \mathcal{L a t}_{2 k_{0}}(\ell), \\
\Delta_{2 k_{1}}= & (\eta(z) \eta(\ell z))^{k_{1}} \in{ }^{0} \mathcal{M}_{k_{1}}^{+}(\ell) \\
& \Phi_{2 k_{2}} \in{ }^{0} \mathcal{M}_{k_{2}}^{-}(\ell)
\end{aligned}
$$

and where $L_{0}$ is given in Table 1.

TABLE 1. Modular forms $\theta_{2 k_{0}}=\Theta_{L_{0}}$ of weight $k_{0}$

\begin{tabular}{|c|c|c|c|}
\hline$\ell$ & $k_{0}$ & $L_{0}$ & $\theta_{2 k_{0}}=\Theta_{L_{0}}$ \\
\hline 1 & 4 & $\mathbf{E}_{8}$ & $1+240 q^{2}+2160 q^{4}+6720 q^{6}+O\left(q^{8}\right)$ \\
2 & 2 & $\mathbf{D}_{4}$ & $1+24 q^{2}+24 q^{4}+96 q^{6}+O\left(q^{8}\right)$ \\
3 & 1 & $\mathbf{A}_{2} \approx\left(\begin{array}{ll}2 & 1 \\
1 & 2\end{array}\right)$ & $1+6 q^{2}+6 q^{6}+O\left(q^{8}\right)$ \\
5 & 2 & $\mathbf{A}_{4}$ & $1+20 q^{2}+30 q^{4}+60 q^{6}+O\left(q^{8}\right)$ \\
7 & 1 & $\left(\begin{array}{ll}2 & 1 \\
1 & 4\end{array}\right)$ & $1+2 q^{2}+4 q^{4}+O\left(q^{8}\right)$ \\
11 & 1 & $\left(\begin{array}{ll}2 & 1 \\
1 & 6\end{array}\right)$ & $1+2 q^{2}+4 q^{6}+O\left(q^{8}\right)$ \\
23 & 1 & $\left(\begin{array}{lll}4 & 1 \\
1 & 6\end{array}\right)$ or $\left(\begin{array}{ll}2 & 1 \\
1 & 12\end{array}\right)$ & $1+2 q^{2}+O\left(q^{8}\right)$ or $1+2 q^{4}+2 q^{6}+O\left(q^{8}\right)$ \\
\hline
\end{tabular}

For a proof, see [BacVen01, §2] and Queb95, §3]. Recall that

$$
\begin{aligned}
\eta(z) & =q^{1 / 12} \prod_{m=1}^{\infty}\left(1-q^{2 m}\right) \\
& =q^{1 / 12}\left(1-q^{2}-q^{4}+O\left(q^{10}\right)\right), \quad q=e^{i \pi z} .
\end{aligned}
$$

For $\ell=1$, the modular forms of the theorem are $\Delta_{24}=\eta(z)^{24}$, whose Fourier coefficients are the Ramanujan numbers,

$$
\Theta_{\mathbf{E}_{8}}=E_{(4)} \quad \text { and } \quad \Phi_{36}=\Delta_{24} E_{(6)},
$$

where $E_{(k)}$ is the Eisenstein series of weight $k$.

Tables 1 to 3 give the theta series $\theta_{2 k_{0}}, \Delta_{2 k_{1}}$, and $\Phi_{2 k_{2}}$ for all possible values of $\ell$. In the third column of Table 1 , the lattice $L_{0}$ is designated either by a root system that generates it (e.g., $\left.\mathbf{E}_{8}\right)$, or by a quadratic form (e.g., $\left.\left(\begin{array}{ll}2 & 1 \\ 1 & 4\end{array}\right)\right)$. In Table $3, P_{k}$ is a suitable element of $\mathcal{H}^{(k)}\left(\mathbb{R}^{n}\right)$, the lattice $L_{4}$ is a unique (up to isometry) 4-dimensional lattice of minimum 4 and determinant $11^{2}$, and the lattices $L_{0}^{(1)}$ and $L_{0}^{(2)}$ are the two lattices given in the column $L_{0}$ of Table 1 . We normalize $\Phi_{2 k_{2}}$ by $\Phi_{2 k_{2}}=q^{2}+O\left(q^{4}\right)$. 
TABLE 2. Modular forms $\Delta_{2 k_{1}}=(\eta(z) \eta(\ell z))^{k_{1}}$ of weight $k_{1}$

\begin{tabular}{|c|c|c|}
\hline$\ell$ & $k_{1}$ & $\Delta_{2 k_{1}}=(\eta(z) \eta(\ell z))^{k_{1}}$ \\
\hline 1 & 12 & $q^{2}-24 q^{4}+252 q^{6}-1472 q^{8}+O\left(q^{10}\right)$ \\
2 & 8 & $q^{2}-8 q^{4}+12 q^{6}+64 q^{8}+O\left(q^{10}\right)$ \\
3 & 6 & $q^{2}-6 q^{4}+9 q^{6}+4 q^{8}+O\left(q^{10}\right)$ \\
5 & 4 & $q^{2}-4 q^{4}+2 q^{6}+8 q^{8}+O\left(q^{10}\right)$ \\
7 & 3 & $q^{2}-3 q^{4}+5 q^{6}-7 q^{8}+O\left(q^{10}\right)$ \\
11 & 2 & $q^{2}-2 q^{4}-q^{6}+2 q^{8}+O\left(q^{10}\right)$ \\
23 & 1 & $q^{2}-q^{4}-q^{6}+O\left(q^{10}\right)$ \\
\hline
\end{tabular}

TABLE 3. Modular forms $\Phi_{2 k_{2}}$ of weight $k_{2}$

\begin{tabular}{|c|c|c|c|}
\hline$\ell$ & $k_{2}$ & $\Phi_{2 k_{2}}$ (definition) & $\Phi_{2 k_{2}}$ (expansion) \\
\hline 1 & 18 & $\Theta_{\mathbf{E}_{8}, P_{14}}$ & $q^{2}-528 q^{4}-4284 q^{6}$ \\
2 & 12 & & $+147712 q^{8}+O\left(q^{10}\right)$ \\
& & $\Theta_{\mathbf{D}_{4}^{2}, P_{8}}-\Theta_{\left(\mathbf{D}_{4}^{2}\right)^{\prime}, P_{8}}$ & $q^{2}-88 q^{4}+252 q^{6}$ \\
3 & 9 & $\Theta_{\mathbf{A}_{2}^{3}, P_{6}}+\Theta_{\left(\mathbf{A}_{2}^{3}\right)^{\prime}, P_{6}}$ & $+64 q^{8}+O\left(q^{10}\right)$ \\
& & & $q^{2}-14 q^{4}+48 q^{6}$ \\
5 & 8 & $\Theta_{L_{0}, P_{6}}+\Theta_{L_{0}^{\prime}, P_{6}}$ & $+68 q^{8}+O\left(q^{10}\right)$ \\
7 & 7 & $\Theta_{L_{0}^{2}, P_{4}}-\Theta_{\left(L_{0}^{2}\right)^{\prime}, P_{4}}$ & $q^{2}-14 q^{4}-48 q^{6}$ \\
& & & $+68 q^{8}+O\left(q^{10}\right)$ \\
11 & 5 & $\Theta_{L_{0} \perp L_{4}, P_{2}}+\Theta_{\left(L_{0} \perp L_{4}\right)^{\prime}, P_{2}}$ & $q^{2}-6 q^{4}-3 q^{6}-14 q^{8}+O\left(q^{10}\right)$ \\
23 & 4 & $\Theta_{L_{0}^{(1)} \perp L_{0}^{(2)}, P_{2}}+\Theta_{\left(L_{0}^{(1)} \perp L_{0}^{(2)}\right)^{\prime}, P_{2}}$ & $q^{2}-2 q^{4}-5 q^{6}-4 q^{8}+O\left(q^{10}\right)$ \\
\hline
\end{tabular}

\section{$\S 5$. Construction of Cubature Formulas USing Shells of Lattices}

Let $G$ be a finite subgroup of $\mathrm{O}(n)$, and let $X_{1}, X_{2}, \ldots, X_{r}$ be nonempty finite subsets of $\mathbb{S}^{n-1}$ such that $G X_{j}=X_{j}$. Consider the set

$$
X:=\bigcup_{j=1}^{r} X_{j} \subseteq \mathbb{S}^{n-1}
$$

Our aim is to find numbers $W_{1}, W_{2}, \ldots, W_{r} \in \mathbb{R}_{>0}$ such that $(X, W)$ is a cubature formula of high strength, where $W$ is the weight function given by

$$
W(x)=\sum_{j: x \in X_{j}} W_{j}
$$


By Proposition $5,(X, W)$ is of strength $t$ if and only if the following conditions on $W_{1}, \ldots, W_{r}$ are satisfied:

$$
\begin{gathered}
\sum_{j=1}^{r} W_{j}\left|X_{j}\right|=1, \\
\sum_{j=1}^{r} W_{j} P\left(X_{j}\right)=0 \quad \text { for all } P \in \mathcal{H}^{(k)}\left(\mathbb{R}^{n}\right)^{G}, 1 \leq k \leq t,
\end{gathered}
$$

where

$$
P\left(X_{j}\right):=\sum_{x \in X_{j}} P(x)
$$

The following statement is fundamental for our analysis.

9. Lemma. Let $G$ be a finite subgroup of $\mathrm{O}(n)$, and let $E \subseteq \mathbb{R}^{n}$ be a discrete set that is invariant by $G$. For pairwise distinct positive numbers $m_{1}, m_{2}, \ldots, m_{r}$, let

$$
X_{j}=\frac{1}{\sqrt{m_{j}}} E_{m_{j}} \subseteq \mathbb{S}^{n-1}, \quad j=1, \ldots, r
$$

where $E_{m}=\{x \in E \mid\langle x \mid x\rangle=m\}$. Let $X=\bigcup_{j=1}^{r} X_{j}$ and $W_{1}, \ldots, W_{r}>0$, and let $k$ be a positive integer.

Assume that there exist formal series

$$
\Theta_{i}=\sum_{m>0} a_{i}(m) q^{m}, \quad i=1, \ldots, N,
$$

such that for every $P \in \mathcal{H}^{(k)}\left(\mathbb{R}^{n}\right)^{G}$ the theta series $\Theta_{E, P}$ is of the form

$$
\Theta_{E, P}=c_{1}(P) \Theta_{1}+c_{2}(P) \Theta_{2}+\cdots+c_{N}(P) \Theta_{N}
$$

for some $c_{i}(P) \in \mathbb{C}$.

Then, for the condition

$$
\sum_{j=1}^{r} W_{j} P\left(X_{j}\right)=0 \quad \text { for all } P \in \mathcal{H}^{(k)}\left(\mathbb{R}^{n}\right)^{G}
$$

to hold, it suffices that

$$
\sum_{j=1}^{r} \frac{a_{i}\left(m_{j}\right)}{m_{j}^{k / 2}} W_{j}=0, \quad i=1, \ldots, N .
$$

Proof. Assume that $(\sharp)$ is true, and let $P \in \mathcal{H}^{(k)}\left(\mathbb{R}^{n}\right)^{G}$. By hypothesis, there exist $c_{1}, \ldots, c_{N} \in \mathbb{C}$ such that

$$
\sum_{m>0} P\left(E_{m}\right) q^{m}=\Theta_{E, P}=\sum_{i=1}^{N} c_{i} \Theta_{i}=\sum_{m>0}\left(\sum_{i=1}^{N} c_{i} a_{i}(m)\right) q^{m} .
$$

Since $P$ is homogeneous of degree $k$, we have $P\left((1 / \sqrt{m}) E_{m}\right)=m^{-k / 2} P\left(E_{m}\right)$. Therefore,

$$
P\left(X_{j}\right)=m_{j}^{-k / 2} P\left(E_{m_{j}}\right)=\sum_{i=1}^{N} c_{i} \frac{a_{i}\left(m_{j}\right)}{m_{j}^{k / 2}},
$$

whence

$$
\sum_{j=1}^{r} W_{j} P\left(X_{j}\right)=\sum_{i=1}^{N} c_{i} \sum_{j=1}^{r} \frac{a_{i}\left(m_{j}\right)}{m_{j}^{k / 2}} W_{j}=0
$$




\section{$\S 6$. Applications}

If not otherwise specified, Lemma 9 is applied with the trivial group $G=\{\mathrm{id}\}$. In this section, we do not give the details for all lattices we have considered, but we have made a selection which reflects most situations that may occur. All cubature formulas we have obtained are listed in $\$ 7$ below.

6.1. The Leech lattice. Let $\Lambda$ be the Leech lattice, namely, a unique (up to isometry) even unimodular lattice of dimension 24 and of minimum 4 . Let $P \in \mathcal{H}^{(2 h)}\left(\mathbb{R}^{24}\right)$. From Proposition 7 and Theorem 8, we deduce (using $\Lambda^{\prime}=\Lambda$ ):

$$
\Theta_{\Lambda, P}= \begin{cases}\Theta_{\mathbf{E}_{8}}^{3}-720 \Delta_{24} & \text { if } P=1, \\ 0 & \text { if } P \in \mathcal{H}^{(2 h)}\left(\mathbb{R}^{24}\right), 2 h=2,4,6,8,10,14, \\ c_{1}(P) \Delta_{24}^{2} & \text { if } P \in \mathcal{H}^{(12)}\left(\mathbb{R}^{24}\right), \\ c_{2}(P) \Theta_{\mathbf{E}_{8}} \Delta_{24}^{2} & \text { if } P \in \mathcal{H}^{(16)}\left(\mathbb{R}^{24}\right),\end{cases}
$$

where $c_{1}$ and $c_{2}$ are linear forms on $\mathcal{H}^{(12)}\left(\mathbb{R}^{24}\right)$ and $\mathcal{H}^{(16)}\left(\mathbb{R}^{24}\right)$, respectively. The first coefficients of these theta series are

$$
\begin{aligned}
\Theta_{\mathbf{E}_{8}}^{3}-720 \Delta_{24} & =1+196560 q^{4}+16752960 q^{6}+O\left(q^{8}\right), \\
\Delta_{24}^{2} & =q^{4}-48 q^{6}+O\left(q^{8}\right), \\
\Theta_{\mathbf{E}_{8}} \Delta_{24}^{2} & =q^{4}+192 q^{6}+O\left(q^{8}\right) .
\end{aligned}
$$

Since $\Theta_{\Lambda, P}=0$ for $P \in \mathcal{H}^{(2 h)}\left(\mathbb{R}^{24}\right), 2 \leq 2 h \leq 10$, it follows from Proposition 4 that every shell of the Leech lattice is a spherical 11-design.

Now, we want to find cubature formulas of higher strengths using the construction of \$5. Consider the set

$$
X=X_{1} \cup X_{2}, \quad \text { where } X_{1}=\frac{1}{\sqrt{4}} \Lambda_{4}, X_{2}=\frac{1}{\sqrt{6}} \Lambda_{6} .
$$

The two sets $X_{1}$ and $X_{2}$ are disjoint. Indeed, if $x \in X_{1} \cap X_{2}$, then $\sqrt{4} x \in \Lambda_{4}$ and $\sqrt{6} x \in \Lambda_{6}$, but $\langle\sqrt{4} x \mid \sqrt{6} x\rangle=2 \sqrt{6}\langle x \mid x\rangle=2 \sqrt{6}$, which is impossible since $\Lambda$ is integral.

We want to find numbers $W_{1}$ and $W_{2}$ such that $(X, W)$ is a cubature of strength 15 (same notation as in 95 ). For this, we have to fulfill the conditions

$$
\begin{gathered}
\sum_{x \in X} W(x)=1 \\
\sum_{x \in X} W(x) P(x)=0 \quad \text { for } P=\mathcal{H}^{(12)}\left(\mathbb{R}^{n}\right) .
\end{gathered}
$$

The first condition is equivalent to $\left|X_{1}\right| W_{1}+\left|X_{2}\right| W_{2}=1$, that is,

$$
196560 W_{1}+16752960 W_{2}=1 \text {. }
$$

By Lemma 9, the second condition is equivalent to

$$
\frac{1}{4^{6}} W_{1}-\frac{48}{6^{6}} W_{2}=0
$$

or $W_{2}=(3 / 4)^{5} W_{1}$. The solution of this set of two linear equations is

$$
W_{1} \approx 2.394 \times 10^{-7}, \quad W_{2} \approx 0.568 \times 10^{-7} .
$$

So, we get a cubature formula of size $196560+16752960=16949520$.

In the same way we can obtain a cubature formula of strength 17 by using the shells of norms 4,6 , and 8 , and a cubature formula of strength 19 by using the shells of norms $4,6,8$, and 10 of the Leech lattice. 
6.2. The Korkin-Zolotarev lattice. Let $\Lambda$ be the Korkin-Zolotarev lattice, which is a unique (up to isometry) even unimodular lattice of dimension 8. It is the lattice generated by the root system of type $\mathbf{E}_{8}$. From Proposition 7 and Theorem 8, we deduce (using $\Lambda^{\prime}=\Lambda$ ):

$$
\Theta_{\Lambda, P}= \begin{cases}\Theta_{\mathbf{E}_{8}} & \text { if } P=1, \\ 0 & \text { if } P \in \mathcal{H}^{(2 h)}\left(\mathbb{R}^{8}\right), 2 h=2,4,6,10, \\ c_{1}(P) \Delta_{24} & \text { if } P \in \mathcal{H}^{(8)}\left(\mathbb{R}^{8}\right), \\ c_{2}(P) \Theta_{\mathbf{E}_{8}} \Delta_{24} & \text { if } P \in \mathcal{H}^{(12)}\left(\mathbb{R}^{8}\right), \\ c_{3}(P) \Phi_{36} & \text { if } P \in \mathcal{H}^{(14)}\left(\mathbb{R}^{8}\right) .\end{cases}
$$

So, by Proposition 4, any shell of the Korkin-Zolotarev lattice is a spherical 7-design, and we can obtain a cubature formula of stength 11 by combining the shells of norms 2 and 4 .

But if we try to obtain a cubature formula of strength 13 of nodes

$$
X=X_{1} \cup X_{2} \cup X_{3}, \quad \text { where } X_{1}=\frac{1}{\sqrt{2}} \Lambda_{2}, X_{2}=\frac{1}{\sqrt{4}} \Lambda_{4}, X_{3}=\frac{1}{\sqrt{6}} \Lambda_{6},
$$

we obtain the weights

$$
W_{1} \approx-0.744 \times 10^{-4}, \quad W_{2} \approx 1.587 \times 10^{-4}, \quad W_{3} \approx 1.005 \times 10^{-4},
$$

with $W_{1}$ negative. A similar problem occurs if we try to use the shells of norms 2, 4 and 8 . Therefore, in order to get a true cubature formula with positive weights, we must use

$$
X=X_{1} \cup X_{2} \cup X_{3}, \quad X_{1}=\frac{1}{\sqrt{2}} \Lambda_{2}, \quad X_{2}=\frac{1}{\sqrt{6}} \Lambda_{6}, \quad X_{3}=\frac{1}{\sqrt{8}} \Lambda_{8} .
$$

We obtain the weights

$$
W_{1} \approx 0.792 \times 10^{-4}, \quad W_{2} \approx 0.457 \times 10^{-4}, \quad W_{3} \approx 0.385 \times 10^{-4} .
$$

Here, we have $X_{1} \subseteq X_{3}$, since $2 x \in \Lambda_{8}$ if $x \in \Lambda_{2}$. Therefore, we obtain a cubature formula of strength 13 of size

$$
|X|=\left|X_{2}\right|+\left|X_{3}\right|=6720+17520=24240
$$

and of weights

$$
W(x)= \begin{cases}W_{2} \approx 0.457 \times 10^{-4} & \text { if } x \in X_{2}, \\ W_{3} \approx 0.385 \times 10^{-4} & \text { if } x \in X_{3} \backslash X_{1}, \\ W_{1}+W_{3} \approx 1.177 \times 10^{-4} & \text { if } x \in X_{1} .\end{cases}
$$

6.3. The Barnes-Wall lattice of dimension 16. Let $\Lambda$ be the Barnes-Wall lattice of dimension 16. It is a 2-modular lattice, that is, $\Lambda^{\prime}:=\sqrt{2} \Lambda$ is equivalent to $\Lambda$, and it is of minimum 4. From Proposition 7 and Theorem 8, we have:

$$
\begin{aligned}
\Theta_{\Lambda, P}+\Theta_{\Lambda^{\prime}, P}= \begin{cases}2 \Theta_{\mathbf{D}_{4}}^{4}-192 \Delta_{16} & \text { if } P=1, \\
0 & \text { if } P \in \mathcal{H}^{(2 h)}\left(\mathbb{R}^{16}\right), 2 h=2,4,6,10, \\
c_{1}(P) \Delta_{16}^{2} & \text { if } P \in \mathcal{H}^{(8)}\left(\mathbb{R}^{16}\right), \\
c_{2}(P) \Delta_{16}^{2} \Theta_{\mathbf{D}_{4}}^{2} & \text { if } P \in \mathcal{H}^{(12)}\left(\mathbb{R}^{16}\right), \\
c_{3}(P) \Phi_{24} \Delta_{16} \Theta_{\mathbf{D}_{4}} & \text { if } P \in \mathcal{H}^{(14)}\left(\mathbb{R}^{16}\right),\end{cases} \\
\Theta_{\Lambda, P}-\Theta_{\Lambda^{\prime}, P}= \begin{cases}0 & \text { if } P \in \mathcal{H}^{(2 h)}\left(\mathbb{R}^{16}\right), 2 h=0,2,4,6,8, \\
c_{4}(P) \Delta_{16}^{2} \Theta_{\mathbf{D}_{4}} & \text { if } P \in \mathcal{H}^{(10)}\left(\mathbb{R}^{16}\right), \\
c_{5}(P) \Phi_{24} \Delta_{16} & \text { if } P \in \mathcal{H}^{(12)}\left(\mathbb{R}^{16}\right),\end{cases}
\end{aligned}
$$


and therefore

$$
\Theta_{\Lambda, P}= \begin{cases}\Theta_{\mathbf{D}_{4}}^{4}-96 \Delta_{16} & \text { if } P=1, \\ 0 & \text { if } P \in \mathcal{H}^{(2 h)}\left(\mathbb{R}^{16}\right), 2 h=2,4,6, \\ c_{6}(P) \Delta_{16}^{2} & \text { if } P \in \mathcal{H}^{(8)}\left(\mathbb{R}^{16}\right), \\ c_{7}(P) \Delta_{16}^{2} \Theta_{\mathbf{D}_{4}} & \text { if } P \in \mathcal{H}^{(10)}\left(\mathbb{R}^{16}\right) .\end{cases}
$$

Every shell of $\Lambda$ is a spherical 7-design, and we obtain a cubature formula of strength 9 by combining two different shells of $\Lambda$, for example $\Lambda_{4}$ and $\Lambda_{6}$. We also obtain a cubature formula of strength 11 of support $\frac{1}{\sqrt{4}}\left(\Lambda \cup \Lambda^{\prime}\right)_{4} \cup \frac{1}{\sqrt{6}}\left(\Lambda \cup \Lambda^{\prime}\right)_{6}$, and a cubature formula of strength 13 of support $\frac{1}{\sqrt{4}}\left(\Lambda \cup \Lambda^{\prime}\right)_{4} \cup \frac{1}{\sqrt{6}}\left(\Lambda \cup \Lambda^{\prime}\right)_{6} \cup \frac{1}{\sqrt{10}}\left(\Lambda \cup \Lambda^{\prime}\right)_{10}$.

6.4. The $\mathbf{D}_{4}$ root lattice. Let $\Lambda$ be the lattice generated by the root system $\mathbf{D}_{4}$. It is a 2-modular lattice. Here, we apply Lemma 9 with

$$
G=\operatorname{Aut}\left(\Lambda \cup \Lambda^{\prime}\right) \text {. }
$$

We now describe $\Lambda, \Lambda^{\prime}$ and $G$. We have

$$
\begin{aligned}
\Lambda & =\left\{x=\left(x_{1}, x_{2}, x_{3}, x_{4}\right) \mid x_{i} \in \mathbb{Z}, x_{1}+x_{2}+x_{3}+x_{4} \in 2 \mathbb{Z}\right\}, \\
\Lambda^{\prime} & =\left\{x=\left(x_{1}, x_{2}, x_{3}, x_{4}\right) \mid x_{i} \in \frac{1}{\sqrt{2}} \mathbb{Z}, x_{i}-x_{j} \in \sqrt{2} \mathbb{Z}\right\} .
\end{aligned}
$$

The group $\operatorname{Aut}(\Lambda)$ contains (actually, is) a reflection group, denoted by $\mathrm{W}\left(\mathbf{F}_{4}\right)$, that is generated by the 24 reflections $x \mapsto x-2 \frac{\langle x \mid \alpha\rangle}{\langle\alpha \mid \alpha\rangle} \alpha$, where $\alpha$ is one of the following vectors:

$$
( \pm 1,0,0,0), \quad( \pm 1, \pm 1,0,0), \quad( \pm 1, \pm 1, \pm 1, \pm 1)
$$

(all choices of signs and all permutations of coordinates). The invariant polynomials of this group are known [Smit95, §7.4, pp. 217-218]; they form a polynomial $\mathbb{C}$-algebra of basis $\left\{h_{2}, h_{6}, h_{8}, h_{12}\right\}$, where

$$
\begin{aligned}
h_{2}= & \operatorname{sym}\left(x_{1}^{2}\right), \\
h_{6}= & \operatorname{sym}\left(x_{1}^{4} x_{2}^{2}\right)-3 \operatorname{sym}\left(x_{1}^{2} x_{2}^{2} x_{3}^{2}\right), \\
h_{8}= & \operatorname{sym}\left(x_{1}^{8}\right)+14 \operatorname{sym}\left(x_{1}^{4} x_{2}^{4}\right)+168 x_{1}^{2} x_{2}^{2} x_{3}^{2} x_{4}^{2}, \\
h_{12}= & \operatorname{sym}\left(x_{1}^{12}\right)+22 \operatorname{sym}\left(x_{1}^{6} x_{2}^{6}\right)+165 \operatorname{sym}\left(x_{1}^{4} x_{2}^{4} x_{3}^{4}\right) \\
& +330 \operatorname{sym}\left(x_{1}^{6} x_{2}^{2} x_{3}^{2} x_{4}^{2}\right)+330 \operatorname{sym}\left(x_{1}^{4} x_{2}^{4} x_{3}^{2} x_{4}^{2}\right),
\end{aligned}
$$

and where

$$
\operatorname{sym}(p)=\frac{1}{\left|\left(\mathfrak{S}_{4}\right)_{p}\right|} \sum_{\sigma \in \mathfrak{S}_{4}} \sigma \cdot p, \quad\left(\mathfrak{S}_{4}\right)_{p}:=\left\{\sigma \in \mathfrak{S}_{4} \mid \sigma \cdot p=p\right\}
$$

(the symmetric group $\mathfrak{S}_{4}$ acts by permuting the coordinates). For our purpose, it is better to choose the basis

$$
\begin{aligned}
H_{2} & :=h_{2}, \quad H_{6}:=8 h_{6}-h_{2}^{3}, \quad H_{8}:=10 h_{8}-7 h_{2}^{4}, \\
H_{12} & :=64 h_{12}-55 h_{8} h_{2}^{2}-176 h_{6}^{2}+220 h_{6} h_{2}^{3}-11 h_{2}^{6} .
\end{aligned}
$$

The orthonormal transformation

$$
T:=\left(\begin{array}{cccc}
-\sqrt{2} / 2 & \sqrt{2} / 2 & 0 & 0 \\
-\sqrt{2} / 2 & -\sqrt{2} / 2 & 0 & 0 \\
0 & 0 & \sqrt{2} / 2 & \sqrt{2} / 2 \\
0 & 0 & -\sqrt{2} / 2 & \sqrt{2} / 2
\end{array}\right)
$$


exchanges $\Lambda$ and $\Lambda^{\prime}$. So, $G=\operatorname{Aut}\left(\Lambda \cup \Lambda^{\prime}\right)$ is generated by $\operatorname{Aut}(\Lambda)$ and $T$. The action of $T$ on the polynomials $H_{k}$ looks like this:

$$
\begin{aligned}
T H_{2} & =H_{2}, \\
T H_{6} & =-H_{6}, \\
T H_{8} & =H_{8}, \\
T H_{12} & =-H_{12} .
\end{aligned}
$$

Therefore, the polynomials invariant under $G$ are linearly generated by the polynomials

$$
H_{2}^{\alpha} H_{6}^{\beta} H_{8}^{\gamma} H_{12}^{\delta}, \quad \beta+\delta \equiv 0 \bmod 2 .
$$

Let $\mathcal{P}^{(k)}\left(\mathbb{R}^{4}\right)$ be the space of homogeneous polynomial functions on $\mathbb{R}^{4}$ of degree $k$. Since $\mathcal{H}^{(k)}\left(\mathbb{R}^{4}\right)$ is the kernel of the surjective $G$-equivariant Laplacian $\Delta: \mathcal{P}^{(k)}\left(\mathbb{R}^{4}\right) \rightarrow$ $\mathcal{P}^{(k-2)}\left(\mathbb{R}^{4}\right)$, we have

$$
\begin{aligned}
d_{k}^{G}: & =\operatorname{dim} \mathcal{H}^{(k)}\left(\mathbb{R}^{4}\right)^{G} \\
& =\operatorname{dim} \mathcal{P}^{(k)}\left(\mathbb{R}^{4}\right)^{G}-\operatorname{dim} \mathcal{P}^{(k-2)}\left(\mathbb{R}^{4}\right)^{G}
\end{aligned}
$$

We can compute

$$
\begin{aligned}
\sum_{k \geq 0} d_{k}^{G} X^{k} & =\frac{1+X^{18}}{\left(1-X^{8}\right)\left(1-X^{12}\right)\left(1-X^{24}\right)} \\
& =1+X^{8}+X^{12}+X^{16}+O\left(X^{18}\right) .
\end{aligned}
$$

So, by Proposition 7 we have

$$
\Theta_{\Lambda, P}+\Theta_{\Lambda^{\prime}, P}= \begin{cases}2 \Theta_{\mathbf{D}_{4}} & \text { if } P=1, \\ 0 & \text { if } P \in \mathcal{H}^{(2 h)}\left(\mathbb{R}^{8}\right)^{G}=\{0\}, \\ & \quad 2 h=2,4,6,10,14, \\ c_{1}(P) \Delta_{16} \Theta_{\mathbf{D}_{4}} & \text { if } P \in \mathcal{H}^{(8)}\left(\mathbb{R}^{8}\right)^{G}, \\ c_{2}(P) \Delta_{16}^{2} \Theta_{\mathbf{D}_{4}}^{3} & \text { if } P \in \mathcal{H}^{(12)}\left(\mathbb{R}^{8}\right)^{G} .\end{cases}
$$

Thus, using Lemma 9 together with Proposition 5, we obtain cubature formulas of strength 11 and 15 by combining two or three shells of $\Lambda \cup \Lambda^{\prime}$. Note however that certain combinations of shells, such as $\frac{1}{\sqrt{2}}\left(\Lambda \cup \Lambda^{\prime}\right)_{2} \cup \frac{1}{\sqrt{4}}\left(\Lambda \cup \Lambda^{\prime}\right)_{4}$, are not possible, because Lemma 5 can provide a degenerate linear equations system. (In the present example, we have indeed $\frac{1}{\sqrt{2}}\left(\Lambda \cup \Lambda^{\prime}\right)_{2}=\frac{1}{\sqrt{4}}\left(\Lambda \cup \Lambda^{\prime}\right)_{4}$.)

6.5. The shorter Leech lattice. Let $\Lambda$ be a unique (up to isometry) odd unimodular integral lattice of dimension 23 and of minimum 3; it is the shorter Leech lattice. As for even ones, the theta series of odd unimodular lattices can be computed; see Pach05. So, we can apply our method to construct cubature formulas from the shells of $\Lambda$.

\section{§7. Cubature formulas obtained by our construction}

The cubature formulas we obtain by our method are summarized in Tables 6 to 14 . These tables involve the lattices listed in Tables 4 and 5 .

In Tables 6 to 14, the column "bound" indicates the lower bound of the size of the cubature formulas of strength $t$ on $\mathbb{S}^{n-1}$ obtained by Linear Programming. The indication in parentheses has the following meaning:

- ( $\mathrm{LP} d)$ refers to the estimate of the Linear Programming bound obtained by the procedure described in Subsection "Numerical estimate of the Linear Programming bound" in $\S 2$, where $2 d$ is the degree of the polynomial used for the estimate. The calculations have been performed with "Maple" software, using the "Optimization" package. Because 
TABLE 4. Even $\ell$-modular lattices involved in Tables 6 to 14

\begin{tabular}{|c|c|c|c|}
\hline$n$ & $\ell$ & Notation & Name \\
\hline 4 & 2 & $D_{4}$ & $\mathbf{D}_{4}$ root lattice \\
\hline 8 & 1 & $\Gamma_{8}$ & Korkin-Zolotarev \\
\hline 12 & 3 & $K_{12}$ & Coxeter-Todd \\
\hline 14 & 3 & $Q_{14}$ & Quebbemann \\
\hline 16 & 2 & $B W_{16}$ & Barnes-Wall \\
\hline 20 & 2 & $N_{20}^{(1)}, N_{20}^{(2)}, N_{20}^{(3)}$ & Nebe \\
\hline 24 & 3 & $N_{24}$ & Nebe \\
\hline 24 & 1 & $\Lambda_{24}$ & Leech \\
\hline
\end{tabular}

TABLE 5. Odd unimodular lattice involved in Table 13

\begin{tabular}{|c|c|c|}
\hline$n$ & Notation & Name \\
\hline 23 & $O_{23}$ & Shorter Leech lattice \\
\hline
\end{tabular}

of limitations in time and memory of our computer, we have not always chosen an optimal $d$, in which case we write (LP $\geq d$ ); similarly, when the bound we have found can almost certainly be improved, we put the sign $\geq$ before it.

- (T) refers to the Delsarte or (Fisher-type) bound; it is the same as (LP $d$ ) when $2 d+1=t$ (the strength).

- When it is known that tight spherical designs with the corresponding parameters $(n, t)$ do not exist, we write (D) to indicate the Delsarte bound increased by 1 .

The columns "set" and "shells" indicate (respectively) the set $E$ and the shells $m_{j}$ used in the construction of $\$ 5$, in accordance with the notation of Lemma 9. The column "size" gives the size of the cubature formula so constructed.

In the case where $n=4$, we have also listed in Table 7 the cubature formulas of smallest known size for fixed values of $t$.

Most spherical designs with $n \leq 24$ described in [BacVen01, Table 3, p. 108] appear also in our tables.

In Table $12, N_{20}$ denotes any of the three lattices $N_{20}^{(1)}, N_{20}^{(2)}$, and $N_{20}^{(3)}$.

TABLE 6. Cubature formulas for $n=4$ (see also Table 7)

\begin{tabular}{|c|c|c|c|c|}
\hline Strength & Set & Shells & Size & Bound \\
\hline 5 & $D_{4}$ & 2 & 24 & 21 (D) \\
\hline 7 & $D_{4} \cup D_{4}^{\prime}$ & 2 & 48 & $42($ LP5) \\
\hline 11 & $D_{4} \cup D_{4}^{\prime}$ & 2,6 & 240 & 120 (LP11) \\
\hline 15 & $D_{4} \cup D_{4}^{\prime}$ & $2,6,10$ & 528 & 267 (LP22) \\
\hline
\end{tabular}


TABLE 7. Best-known cubature formulas for $n=4$

\begin{tabular}{|c|l|c|c|}
\hline Strength & Best-known cubature formula & Size & Bound \\
\hline 3 & Root system $\mathbf{A}_{1}^{4}$ & 8 & $8(\mathrm{~T})$ \\
\hline 5 & Root system $\mathbf{D}_{4}$ & 24 & $21(\mathrm{D})$ \\
\hline 7 & {$[$ HadSlo94 } & 46 & $42(\mathrm{LP} 5)$ \\
\hline 9 & Announced in [HadSlo94] & 86 & $74(\mathrm{LP} 7)$ \\
\hline 11 & Vertices of the 600-cell & 120 & $120(\mathrm{LP} 11)$ \\
\hline 19 & $\begin{array}{l}\text { Vertices of the 600-cell and of } \\
\text { its dual, the 120-cell [Sali75] }\end{array}$ & 720 & $500(\mathrm{LP} \geq 18)$ \\
\hline
\end{tabular}

TABLE 8. Cubature formulas for $n=8$

\begin{tabular}{|c|c|c|c|c|}
\hline Strength & Set & Shells & Size & Bound \\
\hline 7 & $\Gamma_{8}$ & 2 & 240 & $240(\mathrm{~T})$ \\
\hline 11 & $\Gamma_{8}$ & 2,4 & 2400 & $1856(\mathrm{LP} 8)$ \\
\hline 13 & $\Gamma_{8}$ & $2,6,8$ & 24240 & $4361(\mathrm{LP} 12)$ \\
\hline 15 & $\Gamma_{8}$ & $2,4,6,8$ & 26400 & $9190(\mathrm{LP} 16)$ \\
\hline
\end{tabular}

TABle 9. Cubature formulas for $n=12$

\begin{tabular}{|c|c|c|c|c|}
\hline Strength & Set & Shells & Size & Bound \\
\hline 5 & $K_{12}$ & 4 & 756 & $157(\mathrm{D})$ \\
\hline 7 & $K_{12} \cup K_{12}^{\prime}$ & 4 & 1512 & $729(\mathrm{D})$ \\
\hline 9 & $K_{12} \cup K_{12}^{\prime}$ & 6 & 8064 & $2940(\mathrm{LP} 6)$ \\
\hline 11 & $K_{12} \cup K_{12}^{\prime}$ & $4,6,8$ & 50400 & $10604(\mathrm{LP} 7)$ \\
\hline
\end{tabular}

TABLE 10. Cubature formulas for $n=14$

\begin{tabular}{|c|c|c|c|c|}
\hline Strength & Set & Shells & Size & Bound \\
\hline 5 & $Q_{14}$ & 4 & 756 & $211(\mathrm{D})$ \\
\hline 7 & $Q_{14} \cup Q_{14}^{\prime}$ & 4 & 1512 & $1121(\mathrm{D})$ \\
\hline 9 & $Q_{14} \cup Q_{14}^{\prime}$ & 4,8 & 89964 & $4902(\mathrm{LP} 6)$ \\
\hline 11 & $Q_{14} \cup Q_{14}^{\prime}$ & $4,6,8$ & 107436 & $20817(\mathrm{LP} 7)$ \\
\hline
\end{tabular}


TABLE 11. Cubature formulas for $n=16$

\begin{tabular}{|c|c|c|c|c|}
\hline Strength & Set & Shells & Size & Bound \\
\hline 7 & $B W_{16}$ & 4 & 4320 & $1633(\mathrm{D})$ \\
\hline 9 & $B W_{16}$ & 4,6 & 65560 & $7753(\mathrm{D})$ \\
\hline 11 & $B W_{16} \cup B W_{16}^{\prime}$ & 4,6 & 131520 & $37166(\mathrm{LP} 7)$ \\
\hline 13 & $B W_{16} \cup B W_{16}^{\prime}$ & $4,6,10$ & 4555200 & $\geq 146153(\mathrm{LP} 8)$ \\
\hline
\end{tabular}

TABLE 12. Cubature formulas for $n=20$

\begin{tabular}{|c|c|c|c|c|}
\hline Strength & Set & Shells & Size & Bound \\
\hline 5 & $N_{20}$ & 4 & 3960 & $421(\mathrm{D})$ \\
\hline 7 & $N_{20} \cup N_{20}^{\prime}$ & 4 & 7920 & $3081(\mathrm{D})$ \\
\hline 9 & $N_{20} \cup N_{20}^{\prime}$ & 4,6 & 345840 & $17711(\mathrm{D})$ \\
\hline 11 & $N_{20} \cup N_{20}^{\prime}$ & $4,6,8$ & 4527600 & $95309(\mathrm{LP} 7)$ \\
\hline
\end{tabular}

TABLE 13. Cubature formulas for $n=23$

\begin{tabular}{|c|c|c|c|c|}
\hline Strength & Set & Shells & Size & Bound \\
\hline 7 & $O_{23}$ & 3 & 4600 & $4600(\mathrm{~T})$ \\
\hline 9 & $O_{23}$ & 3,5 & 958458 & $29901(\mathrm{D})$ \\
\hline 11 & $O_{23}$ & $3,4,6$ & 6574550 & $166808(\mathrm{LP} 7)$ \\
\hline
\end{tabular}

TABLE 14. Cubature formulas for $n=24$

\begin{tabular}{|c|c|c|c|c|}
\hline Strength & Set & Shells & Size & Bound \\
\hline 5 & $N_{24}$ & 6 & 26208 & $601(\mathrm{D})$ \\
\hline 7 & $N_{24} \cup N_{24}^{\prime}$ & 6 & 52416 & $5201(\mathrm{D})$ \\
\hline 11 & $\Lambda_{24}$ & 4 & 196560 & $196560(\mathrm{~T})$ \\
\hline 15 & $\Lambda_{24}$ & 4,6 & 16969680 & $\geq 6179991(\mathrm{LP} 9)$ \\
\hline 17 & $\Lambda_{24}$ & $4,6,8$ & 415003680 & $\geq 27131261(\mathrm{LP} 13)$ \\
\hline 19 & $\Lambda_{24}$ & $4,6,8,10$ & 5044384800 & $\geq 116303274(\mathrm{LP} 15)$ \\
\hline
\end{tabular}




\section{REFERENCES}

[Andr00] N. N. Andreev, A minimal design of order 11 on the three-dimensional sphere, Mat. Zametki 67 (2000), no. 4, 489-497; English transl., Math. Notes 67 (2000), no. 3-4, 417-424. MR.1769895 (2001g:05020)

[BacVen01] Ch. Bachoc and B. B. Venkov, Modular forms, lattices and spherical designs, Réseaux euclidiens, designs sphériques et formes modulaires, Monogr. Enseign. Math., no 37, Enseign. Math., Geneva, 2001, pp. 87-111. MR.1878746 (2003d:11096)

[BanDam79] E. Bannai and R. Damerell, Tight spherical designs. I, J. Math. Soc. Japan 31 (1979), 199-207. MR0519045 (80b:05014)

[BanDam80] _ Tight spherical designs. II, J. London Math. Soc. (2) 21 (1980), 13-30. MR0576179 (81g:05024)

[BaMuVe02] E. Bannai, A. Munemasa, and B. Venkov, The nonexistence of certain tight spherical designs, Algebra i Analiz 16 (2004), no. 4, 1-23; English transl., St. Petersburg Math. J. 16 (2005), no. 4, 609-625. MR2090848(2005e:05022)

[Bajn91] B. Bajnok, Chebyshev-type quadrature formulas on the sphere, Proceedings of the TwentySecond Southeastern Conference on Combinatorics, Graph Theory, and Computing (Baton Rouge, LA, 1991), Congr. Numer. 85 (1991), 214-218. MR1152134(93a:05038)

[ConSlo99] J. H. Conway and N. J. A. Sloane, Sphere packings, lattices and groups, 3rd ed., Grundlehren Math. Wiss., vol. 290, Springer-Verlag, New York, 1999. MR1662447 (2000b:11077)

[DeGoSe77] P. Delsarte, J.-M. Goethals, and J. J. Seidel, Spherical codes and designs, Geom. Dedicata 6 (1977), 363-388. MR0485471 (58:5302)

[Dick19] L. E. Dickson, History of the theory of numbers. Vol. II: Diophantine analysis, Carnegie Inst., Washington, 1919; reprinted by Chelsea Publ. Co., New York, 1966. MR0245500 (39:6807b)

[Ebel94] W. Ebeling, Lattices and codes. A course partially based on lectures by F. Hirzebruch, Friedr. Vieweg and Sohn, Braunschweig, 1994; 2nd revised ed., 2002. MR1280458 (95c:11084), MR1938666 (2003i:11093)

[GoeSei79] J.-M. Goethals and J. J. Seidel, Spherical designs, Relations Between Combinatorics and Other Parts of Mathematics (Proc. Sympos. Pure Math., Ohio State Univ., Columbus, Ohio, 1978), Proc. Sympos. Pure Math., vol. 34, Amer. Math. Soc., Providence, RI, 1979, pp. 255-272. MR0525330 (82h:05014)

[GoeSei81] _ Cubature formulae, polytopes, and spherical designs, The Geometric Vein, The Coxeter Festschrift, Springer, New York-Berlin, 1981, pp. 203-218. MR0661779 (83k:05033)

[HadSlo94] R. H. Hardin and N. J. A. Sloane, Expressing $\left(a^{2}+b^{2}+c^{2}+d^{2}\right)^{3}$ as a sum of 23 sixth powers, J. Combin. Theory Ser. A 68 (1994), 481-485. MR1297185 (96e:11048)

[HadSlo96] _ McLaren's improved snub cube and other new spherical designs in three dimensions, Discrete Comput. Geom. 15 (1996), 429-441. MR.1384885 (97b:52013)

[HarPac04] P. de la Harpe and C. Pache, Spherical designs and finite group representations (some results of E. Bannai), European J. Combin. 25 (2004), 213-227. MR.2070542 (2005f:05031)

[HarPac05] Cubature formulas, geometrical designs, reproducing kernels, and Markov operators, Infinite Groups: Geometric, Combinatorial and Dynamical Aspects, Progr. Math., vol. 248, Birkhäuser, Basel, 2005, pp. 219-267. MR2195455

[Kupe06a] G. Kuperberg, Numerical cubature using error-correcting codes, SIAM J. Numer. Anal. 44 (2006), no. 3, 897-907 (electronic).

[Kupe06b] , Numerical cubature from Archimedes' hat-box theorem, SIAM J. Numer. Anal. 44 (2006), no. 3, 908-935 (electronic).

[Lehm47] D. H. Lehmer, The vanishing of Ramanujan's function $\tau(n)$, Duke Math. J. 14 (1947), 429-433. MR0021027 (9:12b)

[MartV01] J. Martinet (ed.), Réseaux euclidiens, designs sphériques et formes modulaires. Autour des travaux de B. Venkov, Monogr. Enseign. Math., no 37, Enseign. Math., Geneva, 2001. MR $1881618(2002 \mathrm{~h}: 11062)$

[NikNik02] S. Nikova and V. Nikov, Improvement of the Delsarte bound for $\tau$-designs when it is not the best bound possible, Des. Codes Cryptogr. 28 (2003), no. 2, 201-222. MR.1962806 (2003m:05046)

[Pach05] C. Pache, Shells of selfdual lattices viewed as spherical designs, Internat. J. Algebra Comput. 15 (2005), no. 5-6, 1085-1127. MR2197823

[Queb95] H.-G. Quebbemann, Modular lattices in Euclidean spaces, J. Number Theory 54 (1995), no. 2, 190-202. MR1354045 (96i:11072) 
[Rank77] R. A. Rankin, Modular forms and functions, Cambridge Univ. Press, Cambridge, 1977. MR0498390 (58:16518)

[Sali75] G. N. Salikhov, Cubature formulae for a hypersphere that are invariant with respect to the group of the regular 600-gon, Dokl. Akad. Nauk SSSR 223 (1975), no. 5, 1075-1078; English transl., Soviet Math. Dokl. 16 (1975), no. 4, 1046-1050. MR0408213 (53:11978)

[Serr85] J.-P. Serre, Sur la lacunarité des puissances de $\eta$, Glasgow Math. J. 27 (1985), 203-221 $=$ Euvres, Collected Papers IV. 1985-1998, Springer-Verlag, Berlin, 2000, pp. 66-84, 640. MR0819840 (87e:11066) MR1730973 (2001e:01037)

[SeyZas84] P. D. Seymour and T. Zaslavsky, Averaging sets: A generalization of mean values and spherical designs, Adv. in Math. 52 (1984), 213-240. MR0744857 (85m:05031)

[Smit95] L. Smith, Polynomial invariants of finite groups, Res. Notes Math., No. 6, A. K. Peters, Wellesley, MA, 1995. MR1328644 (96f:13008)

[Sobo62] S. L. Sobolev, Cubature formulas on the sphere which are invariant under transformations of finite rotation groups, Dokl. Akad. Nauk SSSR 146 (1962), no. 2, 310-313; English transl. in Soviet Math. Dokl. 3 (1962). MR0141225 (25:4635)

[Sobo96] S. L. Sobolev and V. Z. Vaskevich, Cubature formulas, Akad. Nauk Sibirsk. Otdel. Inst. Mat., Novosibirsk, 1996; English transl., Math. Appl., vol. 415, Kluwer Acad. Publishers Group, Dordrecht, 1997. MR1463155 (99g:65029a) MR1462617 (99g:65029b)

[Venk84] B. B. Venkov, Even unimodular extremal lattices, Trudy Mat. Inst. Steklov. 165 (1984), 4348; English transl., Proc. Steklov Inst. Math. 1985, no. 3, 47-52. MR0752931(85i:05044)

[VenMar01] B. B. Venkov (notes by J. Martinet), Réseaux et designs sphériques, Réseaux euclidiens, designs sphériques et formes modulaires, Monogr. Enseign. Math., no 37, Enseign. Math., Geneva, 2001, pp. 10-86. MR1878745 (2002m:11061)

[Vile68] N. Ya. Vilenkin, Special functions and the theory of group representations, "Nauka", Moscow, 1965; English transl., Transl. Math. Monogr., vol. 22, Amer. Math. Soc., Providence, RI, 1968. MR0209523(35:420) MR0229863 (37:5429)

[Yudi97] V. A. Yudin, Lower bounds for spherical designs, Izv. Ross. Akad. Nauk Ser. Mat. 61 (1997), no. 3, 213-223; English transl., Izv. Math. 61 (1997), no. 3, 673-683. MR.1478566 (98i:05042)

Section de Mathématiques, Université de Genève, C.P. 64, 1211 Genève 4, Switzerland

E-mail address: Pierre.delaHarpe@math.unige.ch

Section de Mathématiques, Université de Genève, C.P. 64, 1211 Genève 4, Switzerland

E-mail address: Claude.Pache@math.unige.ch

St. Petersburg Branch, Steklov Mathematical Institute, Russian Academy of Sciences, Fontanka 27, St. Petersburg 191023, Russia

E-mail address: bbvenkov@yahoo.com

Received 3/JUN/2005

Originally published in English 